\title{
A NOTE ON SOLUTIONS TO THE WEINER-HOPF EQUATION WITH POSITIVE KERNEL
}

\section{J. MINEKA}

AbSTRACT. For the Wiener-Hopf equation with positive kernel, $n>0$ an integer, either (1) there is a unique solution with bounded $n$th order differences, or (2) there is a unique solution with $n$th order differences approaching zero, or (3) there is no solution with bounded differences. Necessary and sufficient conditions for (1), (2) and (3) are formulated probabilistically.

The positive solution to the Wiener-Hopf equation with positive kernel,

$$
\sum_{y=0}^{\infty} k(y-x) h(y)=h(x), \quad x=0,1,2, \cdots,
$$

has been studied intensively.

Let $\sum_{-\infty}^{\infty} k(x)=1$ and the greatest common divisor of the set of integers $\{x / k(x) \neq 0\}$ be 1 . Let $P\{\}$ be the probability measure for the random walk on the integers with transition probabilities defined by $k(x)$, i.e. if $\Omega=$ $\left\{\left(X_{0}, X_{1}, X_{2}, \cdots\right)\right\}$ is the space of sequences of integers, $P$ is constructed using the relation $P\left\{X_{n+1}=y / X_{n}=x\right\}=k(y-x)$, and an arbitrary initial distribution, $P\left\{X_{0}=x\right\}=p_{0}(x)$. Thus

$$
\begin{aligned}
P\left\{X_{0}=x_{0}, X_{1}=x_{1}, \cdots, X_{n}=x_{n}\right\} \\
=p_{0}\left(x_{0}\right) k\left(x_{1}-x_{0}\right) \cdots k\left(x_{n}-x_{n-1}\right) .
\end{aligned}
$$

For convenience we use the notation $P\left\{A / X_{0}=x\right\}=P_{x}\{A\}$ for any measurable $A$ in $\Omega$.

If

$$
P_{0}\left\{X_{n}>0 \text {, some } n>0\right\}=1
$$

then it is well known that a positive solution to (1) exists, namely

$$
G(x)=1+\sum_{t=1}^{x} g(t)
$$

Received by the editors June 17, 1970 and, in revised form, June 11, 1971 and June 12, 1972.

AMS (MOS) subject classifications (1970). Primary 45E10, 60J15.

Key words and phrases. Wiener-Hopf equation, solutions with bounded differences.

(C) American Mathematical Society 1973 
where

$$
g(x)=\sum_{n=1}^{\infty} P_{0}\left\{X_{m} \leqq 0, \text { for all } m<n, X_{n}=-x\right\}, \text { for } x>0 .
$$

The convergence of this expression can be shown by a simple combinatorial argument (cf. Feller, [1, Chapter XII]): Let $X_{m}^{\prime}=X_{n}-X_{n-m}$, $0 \leqq m \leqq n$. Then the $n$-tuple $\left(X_{1}^{\prime}, X_{2}^{\prime}, \cdots, X_{n}^{\prime}\right)$ has the same joint distribution as $\left(X_{1}, X_{2}, \cdots, X_{n}\right)$, so that

$$
\begin{aligned}
P_{0}\left\{X_{m} \leqq 0, \text { all } m<n, X_{n}=-x\right\} \\
=P_{0}\left\{X_{m}^{\prime} \geqq X_{n}^{\prime}, \text { all } m<n, X_{n}^{\prime}=-x\right\} \\
=P_{0}\left\{X_{m} \geqq X_{n}, \text { all } m<n, X_{n}=-x\right\} .
\end{aligned}
$$

Therefore $g(x)$ can be interpreted as the expectation of the number of minima of the random walk (ladder points) which occur at $-x$. If the random variable $Z$ is defined as the first nonpositive position of the random walk assuming $X_{0}=0$, i.e.

$\{Z=x\}=\left\{\right.$ for some $n>0, X_{m}>0$ for all $m$,

$$
\left.0<m<n ; X_{n}=x \leqq 0\right\},
$$

and if $\left\{Z_{k}\right\}$ is a sequence of independent copies of $Z$, then it follows (cf. $[2$, p. 195]) that

$$
\begin{aligned}
& \sum_{n=1}^{\infty} P_{0}\left\{X_{m} \geqq X_{n}, \text { all } m<n ; X_{n}=-x\right\} \\
&=\sum_{k=1}^{\infty} P_{0}\left\{Z_{1}+Z_{2}+\cdots+Z_{k}=-x\right\} .
\end{aligned}
$$

By the renewal theorem it follows that if $Z$ is defined almost everywhere and if $E|Z|<\infty$, then $g(x) \rightarrow 1 / E|Z|$, while if $P_{0}\{X<0$ for some $n>0\}<1$ so that $Z$ is not almost everywhere defined, or if $E|Z|=\infty$, then $g(x) \rightarrow 0$ as $x \rightarrow \infty$. These properties are useful in discussing the possible solutions of (1).

The question of the uniqueness of the positive solution $G(x)$ to the equation (1) has been answered only for certain classes of kernels $k(x)$. Conditions have been given, for instance, on the moments of $k(x)$, which imply that this solution $G(x)$ is the unique positive solution, apart from multiplication by a constant (cf. Spitzer [2]). Similar results, of course, hold for the adjoint of $(1), k(x)$ being replaced by $k(-x)$ and condition (2) by

$$
P_{0}\left\{X_{n}<0 \text {, some } n>0\right\}=1 .
$$

There are, however, no completely general uniqueness or existence results concerning positive solutions. It is not even known if there is a case in which a positive solution to (1) does not exist (excepting the trivial 
cases when $\sum_{y=0}^{\infty} k(y)=0$ or 1$)$ nor is it known if there is a case in which there are two positive solutions, not constant multiples of each other. The following result, while it does not answer these questions, at least indicates some strong limitations on the possibilities for positive solutions.

For any function $h(x)$, we define a difference operator acting on $h$ by $d(h(x))=h(x+1)-h(x)$, and inductively set $d^{n+1} h(x)=d\left(d^{n}(h(x))\right)$, where $d^{1} h(x)=d(h(x))=d h(x)$. If $d^{n} h(x)$ is uniformly bounded for all $x$, then we will say that $h(x)$ has bounded differences of order $n$.

THEOREM. I. If conditions (2) and ( $\left.2^{\prime}\right)$ hold, then for any $N$, any solution of (1) which has bounded differences of order $N$ is a constant multiple of $G(x)$.

II. If condition (2) holds, but condition (2') is not true, then for any $N$, any solution of (1) whose differences of order $N$ approach zero as $x \rightarrow \infty$ is a constant multiple of $G(x)$.

III. If condition (2) does not hold, then (1) does not have a solution with bounded differences.

Proof. We note first that since $d G(x)=g(x+1)$, and since $g(x) \rightarrow$ $1 / E|Z|$ where $Z$ is the value of the first minimum of the random walk, $G(x)$ always has bounded differences. If $\left(2^{\prime}\right)$ does not hold, then $P_{0}\left\{X_{n} \leqq x\right.$, for some $\left.n\right\} \rightarrow 0$, as $x \rightarrow-\infty$ and hence $g(x) \rightarrow 0$.

We consider first the case $N=1$. Suppose $h(x)$ is a solution to (1) and let $d h(x)=h(x+1)-h(x)$. Set

$$
q_{n}(x, y)=P_{x}\left\{X_{m} \geqq 0 \text { for all } m, 0 \leqq m \leqq n, X_{n}=y\right\} \text {, for } x, y \geqq 0 \text {. }
$$

We note that if $y=0$, then, setting $X_{m}^{\prime}=X_{n}-X_{n-m}$, we have

$$
q_{n}(x, 0)=P_{0}\left\{X_{m}^{\prime} \leqq 0 \text { for all } m, 0 \leqq m \leqq n, X_{n}^{\prime}=-x\right\},
$$

using the same combinatorial argument as before, so that $\sum_{n=1}^{\infty} q_{n}(x, 0)=$ $g(x)$.

Now we write

$$
d h(x)=\sum_{y=0}^{\infty} k(y-x) d h(y)+k(-x-1) h(0) .
$$

Using this relation recursively,

$$
\begin{aligned}
d h(x)= & \sum_{y=0}^{\infty} k(y-x) \sum_{t=0}^{\infty} k(t-y) d h(t) \\
& +\sum_{y=0}^{\infty} k(y-x) k(-y-1) h(0)+k(-x-1) h(0) .
\end{aligned}
$$

$$
d h(x)=\sum_{y=0}^{\infty} q_{n}(x, y) d h(y)+\sum_{m=1}^{n} q_{m}(x+1,0) h(0) .
$$


Since $\sum_{y=0}^{\infty} q_{n}(x, y)=P_{x}\left\{X_{k} \geqq 0\right.$ for $\left.k \leqq n\right\}$ must approach zero as $n \rightarrow \infty$ if $\left(2^{\prime}\right)$ holds, part $I$ is verified, and since in any case $\lim _{n \rightarrow \infty} q_{n}(x, y)=0$, part II is verified. In either of these cases, the first sum on the right of (4) approaches zero as $n \rightarrow \infty$, and we have as a dividend another proof that $G(x)$ is a solution of (1) when condition (2) is satisfied.

If condition (2) is not satisfied, then ( $\left.2^{\prime}\right)$ must be satisfied, and (4) gives us again that $d h(x)=g(x+1) h(0)$ so that $G(x)$ must be a solution. $G(x)$, however, cannot be a solution, since

and

$$
\sum_{y=0}^{\infty} k(y) G(y)=\sum_{y=0}^{\infty} k(y)\left(1+\sum_{t=1}^{y} g(t)\right)
$$

$$
k(y) g(t)=\sum_{n=1}^{\infty} P_{0}\left\{X_{m}<0 \text {, for all } m<n, X_{n}=-t, X_{n+1}=y-t\right\} .
$$

By rearranging the summations in (5) we have that the left hand side is equal to $P_{0}\left\{X_{n}>0\right.$, some $\left.n>0\right\}<1$, contradicting the assertion that $G(x)$ is a solution. This proves part III.

If $N>1$, and (2) and ( $\left.2^{\prime}\right)$ hold, then, for any solution $h(x), h_{1}(x)=$ $h(x)-G(x) h(0)$ is a solution which vanishes at zero. This implies $d h_{1}(x)$ is a solution to (1). Since $G(x)$ has bounded differences, $d h_{1}(x)$ has bounded differences if and only if $h(x)$ has bounded differences of second order. Define inductively $h_{n+1}(x)=d h_{n}(x)-G(x) d h_{n}(0) ; d h_{n+1}(x)=h_{n+1}(x+1)-$ $h_{n+1}(x)$. If $h(x)$ has bounded differences of order $N$, then $d h_{N-1}(x)$ has bounded differences and hence, as a solution to (1), $d h_{N-1}(x)=$ $G(x) d h_{N-1}(0)$. Therefore

$$
h_{N-1}(0) \sum_{t=0}^{x} G(t)=h_{N-1}(x)=d h_{N-2}(x)-G(x) d h_{N-2}(0) .
$$

Now, since $d h_{N-2}(x)$ is a solution, $\sum_{t=0}^{x} G(t)$ must be a solution. But this is easily shown to be untrue. Therefore the solution $h(x)$ could not have had bounded differences of order $N$, unless it was, in fact, a multiple of $G(x)$. Part II is verified similarly.

\section{BIBLIOGRAPHY}

1. W. Feller, An introduction to probability theory and its applications. Vol. II, Wiley, New York, 1966. MR 35 \#1058.

2. F. Spitzer, Principles of random walk, University Series in Higher Math., Van Nostrand, Princeton, N.J., 1964. MR 30 \#1521.

Department of Mathematics, Lehman College, City University of New York, BRONX, NEW YORK 10468 